\title{
Vías para el desarrollo de actividades de innovación y su relación con el desempeño innovador
}

\section{Innovative activity sources and its relation with the innovative performance}

\author{
Ana M M Serrano Bedia $^{1}$ \\ CONCEPCIÓN LÓPEZ FERNÁNDEZ ${ }^{1}$ \\ Gema García PiQueres ${ }^{1}$ \\ Universidad de Cantabria (España)
}

Recibido el 30 de noviembre de 2010, aceptado el 28 de septiembre de 2011

$\mathrm{N}^{\mathrm{o}}$ de clasificación JEL: O31 y O32

DOI: $10.5295 /$ cdg.100272as

\section{Resumen:}

El trabajo estudia el efecto sobre el desempeño innovador de diferentes vías para el desarrollo de actividades de innovación. El estudio empírico utiliza la Encuesta sobre Innovación Tecnológica de las Empresas para el año 2007. Los resultados sugieren que la utilización de una única vía, ya sea interna o externa, tiene un efecto mayoritariamente negativo sobre el desempeño innovador y que los mejores resultados parecen obtenerse de la combinación de todas las vías. Finalmente, las vías cuyo efecto sobre el desempeño parece más independiente de la medida de rendimiento utilizada son interna\&externa, sólo externa e interna\&externa\&cooperación.

Palabras clave:

Vías de innovación, desempeño innovador, empresas activas en innovación.

\begin{abstract}
:
The paper studies the effect of innovative activities sources on innovation performance. For the empirical study we use data from the Encuesta sobre Innovación Tecnológica de las Empresas for the year 2007. The results suggest that the use of only one innovation activity source (internal or external) has a negative effect on innovation performance and that the best innovative performance is obtained when different innovation activities sources are combined. Finally, internal\&external, only external and internal\&external\&cooperation are the innovative activities sources less dependent on the innovative performance measure.
\end{abstract}

Keywords:

Innovation activity sources, innovation performance, firms active in innovation.

\footnotetext{
${ }^{1}$ Facultad de Ciencias Económicas y Empresariales. Departamento de Administración de Empresas (Organización de Empresas). Avda. de los Castros s/n 39005 Santander. serranoa@unican.es; lopezm@unican.es; garciapg@ unican.es
} 


\section{INTRODUCCIÓN}

La innovación se configura como uno de los motores fundamentales en la mejora del bienestar social, el crecimiento y la supervivencia de las empresas a largo plazo (Schumpeter, 1939; Baumol, 2002; Castaño, 2012), entendiéndose como una fuente de competitividad y performance para la empresa (Schmiedeberg, 2008; Galindo et al., 2012). No obstante, y a pesar de los proclamados beneficios de la innovación, la relación entre ésta y una mejora en la performance de la empresa no se produce en todos los casos (Capon et al., 1990; Hart, 1992; Li y Atuahene-Gima, 2001) dado que hay evidencias contrapuestas y no siempre la innovación redunda en mayor performance.

Las decisiones sobre las estrategias de innovación se integran en un proceso de formulación estratégica (Chen y Yuan, 2007) en el que dichas estrategias deberán estar alineadas con la estrategia global de la empresa (Zabala-Iturriagagoitia, 2012), por un lado, y en el que las empresas deben decidir la vía que van a usar para obtener o generar el conocimiento necesario para la innovación, por otro. Tradicionalmente, la literatura ha identificado dos vías principales de desarrollo de innovaciones: "interna" y "externa" (Veugelers y Cassiman, 1999; Cassiman y Veugelers, 2006). Más recientemente, un cuerpo de literatura ha identificado la existencia de una tercera vía, la cooperación con otros agentes (Navarro Arancegui, 2002; Chen y Yuan, 2007), que puede considerarse una forma híbrida (Pisano, 1990) entre las vías interna y externa para el desarrollo de actividades de innovación.

Actualmente, cada vez es menos frecuente que las empresas recurran exclusivamente a las vías internas para el desarrollo de sus actividades de innovación (Fagerberg, 2005; Arango, 2012) sino que tienden a combinar la vía interna con la externa y la cooperación, con el objetivo de poder obtener todas las capacidades necesarias para rentabilizar sus innovaciones (Teece, 1986; Hartung y MacPherson, 2000; Rigby y Zook, 2002). En esta misma línea, diferentes autores sugieren que la interacción entre las diferentes vías de desarrollo de la innovación dentro de la empresa puede constituir la clave para el éxito de sus procesos innovadores (Freeman, 1991; Leiponen, 2005; Tether, 2005).

En consecuencia, el estudio del efecto sobre el desempeño innovador de las diferentes vías elegidas para el desarrollo de las actividades innovadoras constituye el objetivo de este trabajo.

Si bien en la literatura previa existen trabajos que han abordado el estudio de los efectos de las vías de innovación sobre el desempeño innovador, el presente trabajo constituye una aportación a esta corriente científica en los siguientes términos. En primer lugar, dado que los intentos de contrastar estas ideas han dado lugar a resultados contrapuestos, la necesidad de profundizar en el análisis sobre los efectos que las diferentes vías elegidas para la realización de innovaciones tienen sobre el desempeño innovador parece relevante, lo que constituye el principal objetivo de este trabajo. En segundo lugar, que nuestro trabajo introduce, dentro de las limitaciones de los datos empleados, una serie de modificaciones en su planteamiento respecto a otros trabajos previos a fin de poder aportar una visión nueva y más completa de este tema: se plantea el análisis de todas las posibles vías individuales y sus combinaciones para el desarrollo de innovaciones frente a la literatura previa que en la mayoría de los casos no consideraba la vía cooperación, ni la combinación de ésta con la externa. En tercer lugar, se analizan una mayor variedad de medidas de desempeño que en la literatura previa con el fin de conocer en qué medida los diferentes indicadores recogen 
diferentes aspectos relacionados con el rendimiento de las actividades de innovación. Por último, que frente a la mayor parte de la literatura previa, que ha centrado su estudio en la industria manufacturera, la muestra de nuestro trabajo la constituyen tanto empresas manufactureras como de servicios.

Para ello, el presente trabajo se estructura cómo sigue. El segundo apartado aborda la revisión de la literatura sobre el tema objeto de estudio. En el tercer apartado se presentan la fuente de datos, las variables y la metodología. En el cuarto apartado se discuten los resultados mientras que en el último apartado se presentan las conclusiones obtenidas.

\section{REVISIÓN DE LA LITERATURA}

Tal y como se ha señalado en la sección anterior, nuestro objetivo en este trabajo es analizar el efecto sobre el desempeño innovador de la decisión acerca de la vía o vías elegidas para la realización de las actividades de innovación por parte de la empresa. Para ello revisamos la literatura que se ha centrado en los efectos sobre el desempeño de las vías posibles para el desarrollo de actividades de innovación: interna, externa y cooperación, diferenciando los que se producen cuando estas vías se adoptan de manera exclusiva, por un lado, y combinada, por otro.

\subsection{Efectos sobre el desempeño de la utilización de las vías de desarrollo de activi- dades de innovación de manera individual}

Comenzando con la vía interna, su uso para el desarrollo de actividades de innovación puede influir significativamente sobre el desempeño innovador (Katila, 2002; Katila y Ahuja, 2002), una relación positiva que se confirma en trabajos como los de Love y Roper (1999) o Frenz e Ietto-Gillies (2009). Frente a este argumento, otros autores como Chesbrough (2003a, 2003b) a través de su modelo de innovación abierta, (“open innovation”), sugieren que las ventajas derivadas de la elección de la vía interna para el desarrollo de actividades de I+D se han reducido como consecuencia de la mayor movilidad de los trabajadores del conocimiento, lo que dificulta la apropiación y control de los resultados de I+D por parte de la empresa (Laursen y Salter, 2006) y que constituye un fallo de mercado (Pereira y Vence, 2012). Por lo que se refiere a la vía externa, se entiende como adecuada para acceder al conocimiento especializado no disponible internamente y explotar economías de escala asociadas a dicha especialización (Veugelers y Cassiman, 1999), así como para reducir los costes y riesgos del desarrollo interno asociados al mismo (Chen y Yuan, 2007; Huang et al., 2009), todo lo cual redundaría en un mejor rendimiento, una relación positiva que se ha confirmado en trabajos como los de Love y Roper (1999), Stuart (2000), Becker y Dietz, (2002), Francois et al. (2002), Chang (2003), Rogers (2004), Faems et al. (2005) y Frenz e Ietto-Gillies (2009). No obstante, y de acuerdo con los postulados de la Teoría de los Costes de Transacción (Coase, 1937; Williamson, 1985), los altos costes de transacción que surgen como consecuencia de la complejidad, especificidad e incertidumbre asociadas a las actividades de I+D, a los que se añaden los de supervisión y control de las condiciones establecidas en el contrato, reducirían los potenciales beneficios de la vía externa 
(Brockhoff, 1992). Finalmente, y en cuanto se refiere a la cooperación, la literatura pone de manifiesto sus potenciales ventajas en términos de ganancias en eficiencia derivadas de la división del trabajo (Fritsch, 2003), el acceso a conocimiento externo especializado que podría ser difícil o imposible de obtener internamente, al igual que sucedía con la vía externa, el reparto de costes y riesgos (Love y Roper, 2001), el acceso a financiación pública (Veugelers y Cassiman 1999) o el mejor control sobre los spillovers de salida (Cassiman y Veugelers, 2002).

\subsection{Efectos sobre el desempeño de la utilización de manera conjunta de diferentes vías para el desarrollo de actividades de innovación}

El análisis de la relación entre rendimiento innovador y utilización conjunta de varias vías para el desarrollo de actividades de innovación se ha llevado a cabo desde diferentes enfoques teóricos. Así, y desde la teoría de la organización industrial, dos de los principales factores que pueden influir en la relación anteriormente señalada son los spillovers y la capacidad de absorción. Respecto a los spillovers, la mayoría de los modelos teóricos sugieren que las empresas pueden incrementar la efectividad de los spillovers de entrada mediante la inversión en "capacidad de absorción". Desde este punto de vista, el conocimiento procedente del exterior de la empresa no puede convertirse en un input para los procesos innovadores si ésta no desarrolla internamente su propia investigación que le permita absorber, evaluar y utilizar dicho conocimiento externo (Cohen y Levinthal, 1990; Rosenberg, 1990; Kamien y Zang, 2000).

De acuerdo con los planteamientos anteriormente expuestos, la innovación externa y la cooperación tendrían un efecto positivo sobre el desempeño innovador cuando el conocimiento procedente del exterior de la empresa es un input muy relevante de cara al éxito de la innovación, al permitir maximizar los spillovers de entrada. En cuanto al efecto sobre el rendimiento innovador derivado de su utilización conjunta con la I+D interna en presencia de spillovers de entrada algunos autores sugieren, y confirman estadísticamente, la existencia de un efecto negativo (Veugelers, 1997; Jirjahn y Kraft, 2006). La presencia de spillovers de salida también reforzaría este último argumento, al dificultar la apropiación y control de los resultados de I+D por parte de la empresa (Laursen y Salter, 2006).

Frente a este planteamiento, y de acuerdo con el concepto de capacidad de absorción la utilización conjunta de vías interna, externa y cooperación puede contribuir positivamente al rendimiento al permitir a la empresa valorar mejor la calidad de potenciales socios en innovación, a la vez que reforzar su propio atractivo como socio (Veugelers y Cassiman, 1999), poder optar a proyectos de innovación más rentables basándose en la complementariedad de recursos y conocimientos procedentes de diversas vías (Haour, 1992; Arora y Gambardella, 1994) y mejorar la comunicación y coordinación entre las actividades internas y externas, lo que incrementaría la probabilidad de completar con éxito proyectos en colaboración (Bougrain y Haudeville, 2002). Esta relación se ha confirmado empíricamente para el caso de las vías interna y cooperación en Schmiedeberg (2008) y Becker y Peters (2000), las vías interna y externa en Beneito (2006) y Cassiman y Veugelers $(2002 ; 2006)$ y las tres vías conjuntamente en Cassiman y Veugeleres (2002). 
Por otra parte, frente a la noción de capacidad de absorción y su efecto esperado sobre el rendimiento innovador, la literatura (Veugelers y Cassiman, 1999; Laursen y Salter, 2006; Vega-Jurado et al., 2009) también ha puesto de manifiesto la existencia del síndrome NIH (Non Invented Here) (Katz y Allen, 1982). De acuerdo con el mismo, la utilización de vías externas para el desarrollo de innovaciones cuando la empresa realiza un gran esfuerzo en I+D interna puede acarrear cierta resistencia por parte del personal de la propia empresa, al entender que ya posee el monopolio del conocimiento en su campo, lo cual favorecería un efecto negativo de la utilización conjunta de las vías interna y externa sobre el desempeño innovador.

La teoría de los costes de transacción (TCT), por su parte, se ha centrado especialmente en la elección entre desarrollo interno y externo, lo que se conoce como "Make or buy decision" (Veugelers y Cassiman, 1999; Beneito, 2003). De acuerdo con esta teoría, la elección de la vía para el desarrollo de actividades de innovación viene determinada por los costes y riesgos asociados a cada alternativa. Por un lado, la vía externa permite a las empresas eliminar los costes y riesgos asociados al desarrollo interno (Cheng y Yuan, 2007; Huang et al., 2009), los cuales son en general más elevados que los derivados de la adquisición (Beneito, 2003). Por otro, la presencia de elevados niveles de complejidad, especificidad e incertidumbre, como son los asociados a las actividades de I+D, así como la posibilidad de comportamientos oportunistas en las transacciones reducirían los potenciales beneficios de la vía externa, haciendo más eficiente la vía de la jerarquía interna que la del mercado (Williamson, 1985). Estos argumentos apoyarían la hipótesis de un efecto negativo de la combinación de las vías interna y externa sobre el desempeño innovador (Vega-Jurado et al., 2009). En cuanto se refiere a la cooperación, y desde la perspectiva de la TCT, permite compartir costes y riesgos, a la vez que resulta menos vulnerable a los costes de transacción que la vía externa (Veugelers y Cassiman, 1999).

A modo de síntesis de este apartado, la revisión de la literatura efectuada pone de manifiesto la existencia de diferentes argumentos, así como evidencia empírica, que apoyan tanto la hipótesis de un efecto positivo de la combinación de diferentes vías para el desarrollo de actividades de innovación (Becker y Peters, 2000; Beneito, 2006; Cassiman y Veugelers, 2006; Schmiedeberg, 2008; Frenz e Ietto-Gillies, 2009) como de uno negativo (Jirjahn y Kraft, 2006; Laursen y Salter, 2006; Vega-Jurado et al., 2009).

\section{FUENTE DE DATOS, VARIABLES Y METODOLOGÍA}

\subsection{Fuente de datos y muestra de empresas activas en innovación}

El análisis empírico se ha realizado a partir de datos recientes de la Encuesta sobre Innovación Tecnológica de las Empresas para el año 2007 disponibles en el Panel de Innovación Tecnológica (PITEC). El número de empresas recogidas en la Encuesta que pertenecen a los sectores de servicios y manufacturas asciende a $10.814^{1}$. De ellas, $6.807 \mathrm{se}$ clasifican como activas en innovación, y es este grupo el que constituye la muestra que se

\footnotetext{
${ }^{1}$ Al igual que en López (2008) se ha excluido de la muestra a las empresas pertenecientes a los sectores 10 a 14 CNAE (Empresas Extractivas), 40 y 41 (Producción y distribución de electricidad, gas y agua) y 45 (Construcción).
} 
utilizará para la realización del estudio empírico. La distinción entre empresas activas en innovación y las que no lo son se ha realizado en base a la respuesta que dieron éstas a las siguientes preguntas, clasificando como empresa activa en innovación a la que contestó afirmativamente a cualquiera, o las dos, cuestiones siguientes: 1. Si en 2007 la empresa utilizó como vías de innovación para conseguir productos o procesos nuevos o sensiblemente mejorados la I+D interna, la adquisición de $\mathrm{I}+\mathrm{D}$, la adquisición de maquinaria, equipos y hardware o software avanzados, la adquisición de otros conocimientos externos para la innovación, la formación, la introducción de innovaciones en el mercado y/o el diseño, otros preparativos para producción y/o distribución (no incluido I+D); 2. Si durante el periodo 2005-2007 la empresa cooperó con otras empresas o entidades para el desarrollo de actividades de innovación. En cuanto a la muestra seleccionada (empresas activas en innovación), cabe matizar que se analizan éstas y no sólo las empresas innovadoras (aquellas que introdujeron alguna innovación en el mercado), con el objetivo de poder tomar en consideración los procesos innovadores fallidos y no sólo los exitosos -que culminan con la introducción de una innovación en el mercado-.

\subsection{Variables}

Comenzando con las variables dependientes, éstas miden el desempeño innovador y para ello se han tomado diferentes indicadores, debido a que a pesar de la variedad de medidas de desempeño innovador utilizadas en la literatura parece que no existe ningún indicador generalmente aceptado. En muchos casos, la elección viene determinada tanto por los objetivos de la investigación como por la disponibilidad de datos (Beneito, 2006). De hecho, parece evidente que las inconsistencias presentes en los resultados obtenidos en diferentes estudios empíricos se derivan de la dificultad por conseguir que una única medida sea capaz de capturar la complejidad de la innovación (Souitaris, 2002). Para tratar de salvar este inconveniente, Saviotti y Metcalfe (1984) proponen que el uso de varios indicadores de la innovación puede ofrecer una mejor comprensión de la actividad de innovación, por superar el carácter incompleto de cada una de las medidas individuales.

No obstante, hasta el momento no se han realizado muchos análisis que sigan esta propuesta y aborden por tanto, el estudio de la consistencia de los resultados obtenidos con diferentes medidas. Un análisis de este tipo parece de gran interés no tanto para identificar si un indicador es mejor que otro sino, sobre todo, para conocer en qué medida los diferentes indicadores recogen distintos aspectos relacionados con el rendimiento de las actividades de innovación. Por esta razón se han seleccionado tres tipos de medidas del desempeño innovador tomadas a partir la revisión de la literatura (tabla 1).

1. En primer lugar, se toman como variables dependientes medidas del desempeño innovador que clasifican las innovaciones como introducción de productos nuevos para el mercado (INNO MERCADO), o introducción de productos nuevos para la empresa (INNO EMPRESA). De este modo, construimos, en primer lugar, la variable INNO MERCADO como el porcentaje de la cifra de negocios en 2007 debido a innovaciones en bienes y servicios introducidos en el periodo 2005-2007 y que representaron una novedad para el mercado en el que opera la empresa. En segundo lugar, la variable INNO EMPRESA se mide como el porcentaje de la cifra de 
negocios en 2007 debido a innovaciones en bienes o servicios introducidos en el periodo 2005-2007 y que representaron una novedad exclusivamente para la empresa. Ambas medidas de desempeño innovador han sido ampliamente utilizadas en la literatura (Klomp y van Leeuwen, 2001; Love y Roper, 2001; Criscuolo y Haskel, 2003; Monjon y Waelbroeck, 2003; Caloghirou et al., 2004; Faems et al., 2005; Jantunen, 2005; Cassiman y Veuglers, 2006; Marsili y Salter, 2006; Fosfuri y Tribó, 2008; Cetindamar y Ulusoy, 2008; Cockburn et al., 2008; Fosfuri y Tribó, 2008; Schmiedeberg, 2008; Czarnitzki y Wastyn, 2009; Frenz e Ietto-Gillies, 2009; Kirner et al., 2009; Tsai, 2009; Tsai y Wang, 2009; Woerter, 2009).

2. En segundo lugar, se toma una variable de desempeño adicional (EFECTO). Este indicador mide el desempeño innovador en términos de sus "impactos", un tipo de medida que comienza a ser utilizado en algunos trabajos (Souitaris, 2002). Siguiendo a Meeus y Oerlemans (2000) esta variable se ha construido como la media de la importancia que le dieron las empresas a siete ítems diferentes (en una escala de 1-sin importancia a 4-muy importante-) referidos a los efectos de la innovación.

3. En tercer lugar, se tiene como medida de desempeño innovador la introducción de innovaciones en el mercado, diferenciando entre los distintos tipos de innovación obtenida: producto y proceso. En este caso se construyen dos variables dependiendo de si la innovación introducida es en producto (INNOPROD) o en proceso (INNOPROC). Estas variables son similares a las utilizadas en Chang (2003), Knoben (2009), Vega-Jurado et al. (2009) y Weterings y Boschma (2009). INNOPROD y INNOPROC miden la proporción de empresas de cada sector que introdujeron innovaciones en producto o proceso respectivamente, durante el periodo de estudio.

Continuando con las variables independientes, éstas indican las diferentes vías para el desarrollo de innovaciones utilizadas por las empresas (SOLO INTERNA, SOLO EXTERNA, INTERNA\&EXTERNA, INTERNA\&COOP, EXTERNA\&COOP, INTERNA\&EXTERNA\&COOP).

En tercer lugar, en el estudio se han introducido un conjunto de variables de control, que miden características de las empresas, utilizadas en trabajos previos, y que pueden influir en la relación entre las vías para el desarrollo de innovaciones y el desempeño innovador (SECTOR, TAMAÑO, GRUPO, MERGEO, REMUSUP).

Por último, en el estudio empírico se utiliza un cuarto grupo de variables específicas para la realización de la corrección Heckman (1979). Puesto que la muestra de estudio la constituyen únicamente las empresas activas en innovación, para controlar por el posible sesgo en las estimaciones se ha llevado a cabo dicha corrección. Este modelo consiste en una estimación en dos etapas. En primer lugar se realiza una regresión sobre la variable de pertenencia o no al grupo de empresas activas en innovación, de la que se obtiene la inversa del ratio Mills, que se introduce en la regresión del desempeño innovador como variable independiente. De este modo, la nueva estimación será robusta ante la presencia del posible sesgo. En concreto el conjunto de variables utilizadas en la primera son: NONECINNO, OBSREC, FDEMANDA, COSTE, TAMAÑO, GRUPO, MERGEO, REMUSUP y las dummies sectoriales.

La tabla 1 recoge la descripción de la construcción de todas las variables utilizadas en el estudio empírico. 
Tabla 1

Resumen de la construcción de las variables utilizadas en el estudio empírico

\begin{tabular}{|c|c|c|c|}
\hline & Variables & Construcción & Trabajos previos \\
\hline & $\begin{array}{l}\text { INNO } \\
\text { MERCADO }\end{array}$ & $\begin{array}{l}\text { Porcentaje de la cifra de negocios en } 2007 \text { debido a } \\
\text { innovaciones en bienes y servicios introducidos en el } \\
\text { periodo 2005-2007 y que representaron una novedad } \\
\text { para el mercado en el que opera la empresa }\end{array}$ & $\begin{array}{l}\text { Klomp y van Leeuwen (2001); } \\
\text { Love y Roper (2001); Criscuolo } \\
\text { y Haskel (2003); Monjon y Wae- } \\
\text { lbroeck (2003); Caloghirou et al. } \\
\text { (2004); Faems et al. (2005); Jantu- } \\
\text { nen (2005); Cassiman y Veuglers } \\
\text { (2006); Marsili y Salter (2006); } \\
\text { Cetindamar y Ulusoy, (2008); Coc- } \\
\text { kburn et al. (2008); Fosfuri y Tribó } \\
\text { (2008); Schmiedeberg (2008); } \\
\text { Czarnitzki y Wastyn (2009); Frenz } \\
\text { e Ietto-Gillies (2009); Kirner et al. } \\
\text { (2009); Tsai (2009); Tsai y Wang } \\
\text { (2009); Woerter (2009) }\end{array}$ \\
\hline 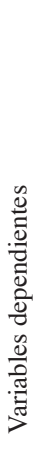 & $\begin{array}{l}\text { INNO } \\
\text { EMPRESA }\end{array}$ & $\begin{array}{l}\text { Porcentaje de la cifra de negocios en } 2007 \text { debido a } \\
\text { innovaciones en bienes o servicios introducidos en el } \\
\text { periodo 2005-2007 y que representaron una novedad } \\
\text { exclusivamente para la empresa }\end{array}$ & $\begin{array}{l}\text { Klomp y van Leeuwen (2001); } \\
\text { Love y Roper (2001); Criscuolo } \\
\text { y Haskel (2003); Monjon y Wae- } \\
\text { lbroeck (2003); Caloghirou et al. } \\
\text { (2004); Faems et al. (2005); Jantu- } \\
\text { nen (2005); Cassiman y Veuglers } \\
\text { (2006); Marsili y Salter (2006); } \\
\text { Cetindamar y Ulusoy, (2008); Coc- } \\
\text { kburn et al. (2008); Fosfuri y Tribó } \\
\text { (2008); Schmiedeberg (2008); } \\
\text { Czarnitzki y Wastyn (2009); Frenz } \\
\text { e Ietto-Gillies (2009); Kirner et al. } \\
\text { (2009); Tsai (2009); Tsai y Wang } \\
\text { (2009); Woerter (2009) }\end{array}$ \\
\hline
\end{tabular}

Media de la importancia que le dieron las empresas (en un escala de 1-sin importancia- a 4-muy importante-) a los efectos de la innovación en términos de: una gama mas amplia de bienes o servicios, penetración en nuevos mercados, mayor calidad de los EFECTO bienes o servicios, mayor flexibilidad en la producción o la prestación de servicios, mayor capacidad de producción o prestación de servicios, menores costes laborales por unidad producida, menos materiales y energía por unidad producida, menor impacto medioambiental o mejora en la salud y la seguridad y cumplimiento de los requisitos normativos 


\begin{tabular}{|c|c|c|c|}
\hline & Variables & Construcción & Trabajos previos \\
\hline \multirow{7}{*}{ 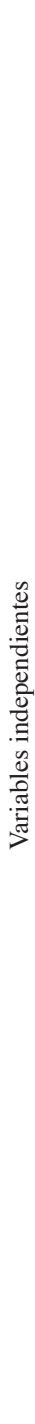 } & $\begin{array}{l}\text { SOLO } \\
\text { INTERNA }\end{array}$ & $\begin{array}{l}\text { Variable dummy que toma valor } 1 \text { si la empresa } \\
\text { desarrolla exclusivamente actividades innovadoras } \\
\text { a través de la vía interna: I+D interna, actividades } \\
\text { para la introducción de innovaciones en el mercado } \\
\text { o diseño y otros preparativos para la producción y/o } \\
\text { distribución, y } 0 \text { en caso contrario }\end{array}$ & $\begin{array}{l}\text { Veugelers (1997); Belderbos et al. } \\
\text { (2004a;b); Veugelers y Cassiman } \\
\text { (2005); Cassiman y Veugelers } \\
\text { (2006) }\end{array}$ \\
\hline & $\begin{array}{l}\text { SOLO } \\
\text { EXTERNA }\end{array}$ & $\begin{array}{l}\text { Variable dummy que toma valor } 1 \text { si la empresa } \\
\text { únicamente utiliza la vía externa para el desarrollo } \\
\text { de sus actividades de innovación, en particular } \\
\text { cuando recurre a la: adquisición de servicios de } \\
\text { I+D, maquinaria, equipos y hardware o software } \\
\text { avanzados o de otros conocimientos externos para } \\
\text { la innovación, y } 0 \text { en caso contrario. }\end{array}$ & $\begin{array}{l}\text { Veugelers (1997); Belderbos et al. } \\
\text { (2004a;b); Veugelers y Cassiman } \\
\text { (2005); Cassiman y Veugelers } \\
\text { (2006) }\end{array}$ \\
\hline & $\begin{array}{l}\text { INTERNA\& } \\
\text { EXTERNA }\end{array}$ & $\begin{array}{l}\text { Variable dummy que toma valor } 1 \text { si la empresa } \\
\text { combina las vías interna y externa para el desarrollo } \\
\text { de innovaciones y } 0 \text { en caso contrario. }\end{array}$ & $\begin{array}{l}\text { Veugelers (1997); Belderbos et al. } \\
\text { (2004a;b); Veugelers y Cassiman } \\
\text { (2005); Cassiman y Veugelers } \\
\text { (2006) }\end{array}$ \\
\hline & $\begin{array}{l}\text { INTERNA\& } \\
\text { COOP }\end{array}$ & $\begin{array}{l}\text { Variable dummy que toma valor } 1 \text { si la empresa } \\
\text { combina las vías internas y la cooperación con otras } \\
\text { empresas y/o instituciones para el desarrollo de } \\
\text { actividades de innovación y } 0 \text { en caso contrario }\end{array}$ & $\begin{array}{l}\text { Veugelers (1997); Belderbos et al. } \\
\text { (2004a;b); Veugelers y Cassiman } \\
\text { (2005); Cassiman y Veugelers } \\
(2006)\end{array}$ \\
\hline & $\begin{array}{l}\text { EXTERNA\& } \\
\text { COOP }\end{array}$ & $\begin{array}{l}\text { Variable dummy que toma valor } 1 \text { si la empresa } \\
\text { combina las vías externa y la cooperación para el } \\
\text { desarrollo de actividades de innovación y } 0 \text { en caso } \\
\text { contrario. }\end{array}$ & $\begin{array}{l}\text { Veugelers (1997); Belderbos et al. } \\
\text { (2004a;b); Veugelers y Cassiman } \\
\text { (2005); Cassiman y Veugelers } \\
\text { (2006) }\end{array}$ \\
\hline & $\begin{array}{l}\text { INTERNA\& } \\
\text { EXTERNA\& } \\
\text { COOP }\end{array}$ & $\begin{array}{l}\text { Variable dummy que toma valor } 1 \text { si la empresa } \\
\text { combina las vías interna, externa y cooperación } \\
\text { para el desarrollo de actividades de innovación y } 0 \\
\text { en caso contrario. }\end{array}$ & $\begin{array}{l}\text { Veugelers (1997); Belderbos et al. } \\
\text { (2004a;b); Veugelers y Cassiman } \\
\text { (2005); Cassiman y Veugelers } \\
\text { (2006) }\end{array}$ \\
\hline & OTROS & $\begin{array}{l}\text { Variable dummy que toma valor } 1 \text { si la empresa de- } \\
\text { sarrolla exclusivamente formación interna o externa } \\
\text { de su personal como actividad de innovación y } 0 \\
\text { en caso contrario. Al no diferenciar la Encuesta de } \\
\text { Innovación Tecnológica entre empresas que realizan } \\
\text { formación interna y las que llevan a cabo formación } \\
\text { externa no resulta posible asignar esta actividad } \\
\text { como exclusivamente interna o exclusivamente } \\
\text { externa. Por ello se ha creado esta variable que en } \\
\text { el estudio empírico se ha tomado como categoría de } \\
\text { referencia }\end{array}$ & $\begin{array}{l}\text { Veugelers (1997); Belderbos et al. } \\
\text { (2004a;b); Veugelers y Cassiman } \\
\text { (2005); Cassiman y Veugelers } \\
\text { (2006) }\end{array}$ \\
\hline
\end{tabular}


Vías para el desarrollo de actividades de innovación y su relación con el desempeño innovador

\begin{tabular}{|c|c|c|c|}
\hline & Variables & Construcción & Trabajos previos \\
\hline \multirow{5}{*}{ 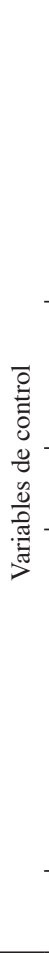 } & SECTOR & $\begin{array}{l}8 \text { variables dummy (CAS1, CAS2, CAS3, CAS4, } \\
\text { CAS5, CAS6, CAS7 y CAS8) que indican el sector } \\
\text { de actividad al que pertenece la empresa. Cada una } \\
\text { de estas variables dummy hace referencia a una de } \\
\text { las categorías sectoriales identificadas en la taxono- } \\
\text { mía de Castellacci (2008) } \text {, y y toman valor } 1 \text { en el } \\
\text { caso de que la empresa pertenezca a esa clasificación } \\
\text { sectorial y } 0 \text { en caso contrario. En este caso la varia- } \\
\text { ble CAS8 es la categoría de referencia. }\end{array}$ & \\
\hline & TAMAÑO & Número de empleados & $\begin{array}{l}\text { Beneito (2006); Jirjahn y Kraft } \\
\text { (2006); Laursen y Salter (2006); } \\
\text { Schmiedeberg (2008); Frenz y } \\
\text { Letto-Gilies (2009) }\end{array}$ \\
\hline & GRUPO & $\begin{array}{l}\text { Variable dummy que toma valor } 1 \text { si la empresa per- } \\
\text { tenece a un grupo de empresas y } 0 \text { en caso contrario. }\end{array}$ & Frenz y Letto-Gilies (2009) \\
\hline & MERGEO & $\begin{array}{l}\text { Esta variable se ha construido a partir de la informa- } \\
\text { ción dada por las empresas acerca de si operan en } \\
\text { mercados locales/autonómicos, nacionales, países de } \\
\text { la UE y/o en todos los demás países. A partir de esta } \\
\text { información, la variable MERGEO se ha construido } \\
\text { como la suma de las respuestas dadas por la empresa } \\
\text { respecto a las cuestiones anteriores, de manera que } \\
\text { las empresas con mayor puntuación serán las empre- } \\
\text { sas más globales o que en mayor número de zonas } \\
\text { geográficas operan. }\end{array}$ & $\begin{array}{l}\text { Laursen y Salter (2006); Frenz y } \\
\text { Letto-Gilies (2009) }\end{array}$ \\
\hline & REMUSUP & $\begin{array}{l}\text { Porcentaje de personal remunerado con educación } \\
\text { superior sobre el total del número de empleados. }\end{array}$ & $\begin{array}{l}\text { Jirjahn y Kraft (2006); Schmiede- } \\
\text { berg (2008) }\end{array}$ \\
\hline \multirow{4}{*}{ 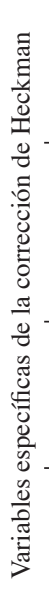 } & $\begin{array}{l}\text { NONECIN- } \\
\text { NO }\end{array}$ & $\begin{array}{l}\text { Importancia que le dieron las empresas a la exis- } \\
\text { tencia de innovaciones previas como barrera a la } \\
\text { innovación en una escala de } 1 \text { (sin importancia) a } 4 \\
\text { (muy importante). }\end{array}$ & Veugelers y Cassiman (1999) \\
\hline & OBSREC & $\begin{array}{l}\text { Media de la importancia que le dieron las empresas a } \\
\text { la falta de personal cualificado, la falta de informa- } \\
\text { ción sobre tecnología y la falta de información sobre } \\
\text { mercados (medidas en escalas de } 1 \text { (sin importancia) } \\
\text { a } 4 \text { (muy importante)) como barreras a la innovación. }\end{array}$ & $\begin{array}{l}\text { Veugelers y Cassiman (1999); Veu- } \\
\text { gelers y Cassiman (2005) }\end{array}$ \\
\hline & $\begin{array}{l}\text { FDEMAN- } \\
\text { DA }\end{array}$ & $\begin{array}{l}\text { Importancia que le dieron las empresas a la falta de } \\
\text { demanda de innovaciones como barrera a la innova- } \\
\text { ción en una escala de } 1 \text { (sin importancia) a } 4 \text { (muy } \\
\text { importante). }\end{array}$ & Cassiman y Veugelers (2006) \\
\hline & COSTE & $\begin{array}{l}\text { Importancia que le dieron las empresas a los costes } \\
\text { de innovación elevados como barrera a la innova- } \\
\text { ción en una escala de } 1 \text { (sin importancia) a } 4 \text { (muy } \\
\text { importante). }\end{array}$ & $\begin{array}{l}\text { Veugelers y Cassiman (1999); } \\
\text { Veugelers y Cassiman (2005); } \\
\text { Cassiman y Veugelers (2006) }\end{array}$ \\
\hline
\end{tabular}

FUENTE: Elaboración propia ${ }^{2}$

${ }^{2}$ CAS1: empresas de servicios intensivas en conocimiento; CAS2: proveedores especializados de manufacturas; CAS3: manufacturas de base científica; CAS4: manufacturas intensivas en escala; CAS5: servicios de infraestructuras de redes (categoría de referencia); CAS6: servicios de infraestructuras físicas; CAS7: bienes dominados por los proveedores; CAS8: servicios dominados por los proveedores. 


\subsection{Metodología}

Para alcanzar los dos objetivos planteados en nuestro estudio usamos diferentes metodologías en función de la variable dependiente en cada caso.

Para estimar el efecto de la combinación de actividades de innovación en las variables INNO MERCADO, INNO EMPRESA y EFECTO, el estudio empírico se basará en un análisis de regresión lineal por MCO sobre la variable dependiente. El modelo teórico de regresión lineal se especifica como sigue:

$$
\begin{gathered}
y_{i}=\beta_{1}+\beta_{2} x_{2 i}+\beta 3 x_{3 i}+\ldots \ldots \ldots+\beta_{k} x_{k i}+\mu_{i} \\
\mathrm{I}=1,2, \ldots, \mathrm{I}
\end{gathered}
$$

La estimación MCO consiste en utilizar como estimación de los parámetros, aquella combinación de $\beta_{1}, \beta_{2}, \ldots \ldots \beta_{\mathrm{k}}$ que minimice los errores.

En cuanto a la estimación para las variables INNOPROD e INNOPROC la metodología se basa en un modelo de regresión logit, técnica estadística apropiada cuando la variable dependiente es categórica y tiene sólo dos grupos, y se puede expresar de la siguiente manera:

$$
Y_{i}=\left\{\begin{array}{l}
1 \operatorname{Prob}\left(Y_{i}=1\right)=P_{i} \\
0 \operatorname{Prob}\left(Y_{i}=0\right)=1-P_{i}
\end{array}\right.
$$

En los modelos logit se asume que las perturbaciones son homocedásticas y no autocorrelacionadas. Además, no se adopta el supuesto de linealidad entre la variable dependiente y las explicativas, cuya relación es de naturaleza no lineal, si bien es cierto, que existe una relación lineal entre el logaritmo del cociente de probabilidades y las variables explicativas (Uriel y Aldás, 2005):

$$
\operatorname{Ln}\left[\frac{P_{i}}{1-P_{i}}\right]=\mathrm{Z}_{i}=\beta_{1}+\sum_{j=2}^{k} \beta_{j} X_{j}
$$


Vías para el desarrollo de actividades de innovación y su relación con el desempeño innovador

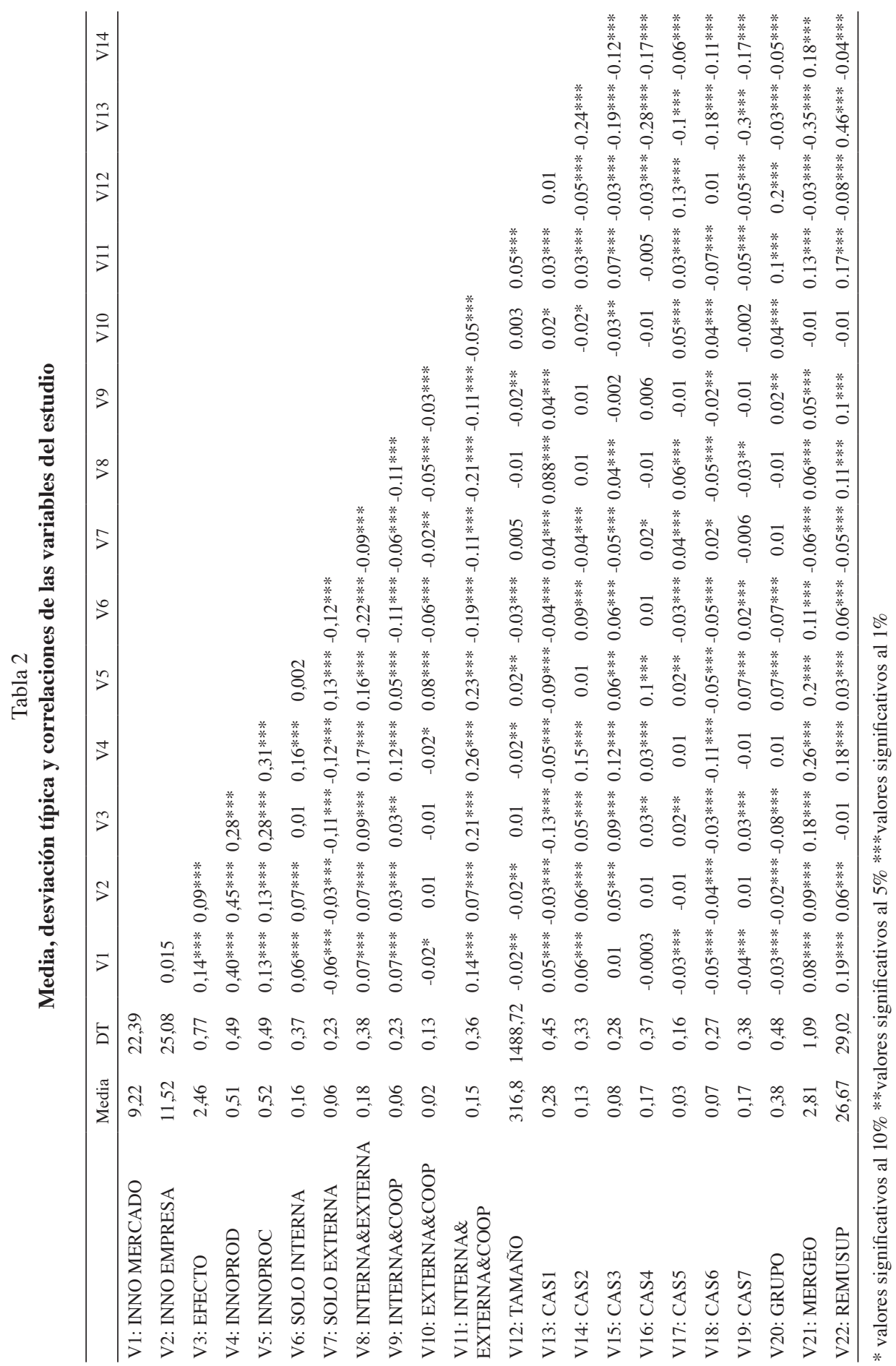


Tabla 2

Media, desviación típica y correlaciones de las variables del estudio (continuación)

\begin{tabular}{lccccccc}
\hline & $\mathrm{V} 15$ & $\mathrm{~V} 16$ & $\mathrm{~V} 17$ & $\mathrm{~V} 18$ & $\mathrm{~V} 19$ & $\mathrm{~V} 20$ & $\mathrm{~V} 21$ \\
\hline V16: CAS4 & $-0.14 * * *$ & & & & & & \\
V17: CAS5 & $-0.05 * * *$ & $-0.07 * * *$ & & & & & \\
V18: CAS6 & $-0.08 * * *$ & $-0.13 * * *$ & $-0.04 * *$ & & & & \\
V19: CAS7 & $-0.14 * * *$ & $-0.21 * * *$ & $-0.07 * * *$ & $-0.13 * * *$ & & & \\
V20: GRUPO & $0.04 * * *$ & $0.02 * *$ & $0.1 * * *$ & $0.05 * * *$ & $-0.04 * * *$ & & \\
V21: MERGEO & $0.16 * * *$ & $0.14 * * *$ & $-0.08 * * *$ & $-0.02 * *$ & $0.14 * * *$ & $0.12 * * *$ & \\
V22: REMUSUP & $0.02 * *$ & $-0.21 * * *$ & $0.05 * * *$ & $-0.08^{* * * *}$ & $-0.2 * * *$ & $-0.04 * * *$ & $-0.05^{* * *}$ \\
\hline
\end{tabular}

$*$ valores significativos al $10 \% * *$ valores significativos al $5 \% * * *$ valores significativos al $1 \%$

\section{RESULTADOS EMPÍRICOS}

Comenzamos este apartado con las frecuencias de las vías de innovación de las empresas españolas (Tabla 3).

Tabla 3

Frecuencias de las combinaciones de las actividades de innovación

\begin{tabular}{lcccccc}
\hline \multicolumn{1}{c}{ Vías de innovación } & $\begin{array}{c}\text { Total de la } \\
\text { muestra }(\%)\end{array}$ & $\begin{array}{c}\text { INNO } \\
\text { MERCA- } \\
\text { DO* }\end{array}$ & $\begin{array}{c}\text { INNO } \\
\text { EMPRESA* }\end{array}$ & EFECTO** & $\begin{array}{c}\text { INNOPROD } \\
(\%) * * *\end{array}$ & $\begin{array}{c}\text { INNOPROC } \\
(\%) * * *\end{array}$ \\
\hline SOLO INTERNA & $1793(26,34)$ & 12,49 & 15,34 & 2,47 & 68 & 52 \\
SOLO EXTERNA & $505(7,42)$ & 3,82 & 9,24 & 2,19 & 29 & 80 \\
INTERNA\&EXTERNA & $1609(23,64)$ & 13,29 & 16,42 & 2,63 & 72 & 71 \\
INTERNA\&COOP & $625(9,18)$ & 15,93 & 14,99 & 2,53 & 75 & 62 \\
EXTERNA\&COOP & $142(2,09)$ & 6,12 & 15,03 & 2,42 & 47 & 85 \\
INTERNA\&EXTERNA & $1690(24,83)$ & 16,56 & 15,79 & 2,79 & 81 & 78 \\
\& COOP & $443(6,51)$ & 11,26 & 13,75 & 2,63 & 68 & 69 \\
OTROS & & & & & 63 \\
\hline
\end{tabular}

* Las columnas con los valores de INNO MERCADO e INNO EMPRESA presentan los valores medios del porcentaje de la cifra de negocios en 2007 debido a innovaciones que supusieron una novedad para el mercado o la empresa respectivamente para cada vía de desarrollo de actividades de innovación.

** La columna con el valor de EFECTO presenta el valor medio de la variable EFECTO para cada vía de innovación.

*** Las columnas con los valores de INNOPROD e INNOPRC presentan el porcentaje de empresas que introdujeron innovaciones de producto y proceso respectivamente para cada vía de desarrollo de actividades de innovación. 
La vía de desarrollo mas usada es SOLO INTERNA (26,34\%), seguida de INTERNA\&EXTERNA\&COOP $\quad(24,83 \%), \quad$ INTERNA\&EXTERNA $\quad(23,64 \%) \quad$ e INTERNA\&COOP $(9,18 \%)$. Estos datos revelan que las empresas españolas activas en innovación lo son mayoritariamente por la vía interna, tanto de forma exclusiva como combinada con otras $(83,99 \%$ del total). Si se relacionan las vías de desarrollo de innovaciones con las diferentes medidas de performance, se pueden extraer los siguientes comentarios. Comenzando con la variable INNO MERCADO los datos de la tabla 3 sugieren que la vía más utilizada para el desarrollo de este tipo de innovaciones es la INTERNA\&EXTERNA\&COOP seguida por la vía INTERNA\&COOP, INTERNA\&EXTERNA y SOLO INTERNA. Parece por tanto, que el desarrollo de innovaciones que suponen una novedad para el mercado se lleva a cabo a través principalmente de la vía interna bien sola o en cualquiera de sus posibles combinaciones. Continuando con la medida de desempeño INNO EMPRESA, los datos de la tabla 3 relacionan este tipo de innovaciones con el uso de las vías INTERNA\&EXTERNA, INTERNA\&EXTERNA\&COOP, SOLO INTERNA, EXTERNA\&COOP e INTERNA\& COOP. Por lo tanto, al igual que para INNO MERCADO, el desarrollo de innovaciones que suponen una novedad para la empresa se relaciona principalmente con la vía interna (sola o combinada), si bien en este caso, un porcentaje significativo de empresas $(15,03 \%)$ se apoya también en la vía EXTERNA\&COOP, sin necesitar por tanto el uso de la vía interna. Respecto de la medida de desempeño EFECTO, la tabla 3 indica que las empresas que adoptan la vía INTERNA\&EXTERNA\&COOP son las que mejores resultados obtienen en términos de los impactos de sus innovaciones, seguidas de las que utilizan la vía INTERNA\&EXTERNA, OTROS e INTERNA\&COOP. Por lo que a la medida de desempeño INNOPROD se refiere, la tabla 3 sugiere que para el desarrollo de innovaciones de producto las empresas se apoyan principalmente en la vía INTERNA\&EXTERNA\&COOP, seguida de la INTERNA\&COOP, INTERNA\&EXTERNA. Sin embargo, para el desarrollo de innovaciones de proceso (INNOPROCESO), la vía más utilizada parece ser la EXTERNA\&COOP, seguida de la SOLO EXTERNA y la INTERNA\&EXTERNA\&COOP. Parece por tanto, que para el desarrollo de innovaciones de proceso, las empresas utilizan menos la vía interna que para el caso de cualquiera de las otras cuatro medidas de desempeño innovador, en las que la utilización de la vía interna tiene mayor peso.

A continuación se exponen los resultados de los análisis de regresión desarrollados (tablas 4 y 5). En primer lugar, los resultados muestran que solo para las estimaciones de las variables EFECTO e INNOPROD es posible señalar que existe un cierto sesgo, si bien los resultados principales en cuanto al efecto de las vías de innovación sobre el desempeño innovador no se ven modificados.

En cuanto a los resultados, comenzado con la vía de desarrollo SOLO INTERNA los resultados señalan un efecto negativo sobre las variables EFECTO e INNOPROC. Este resultado contrasta con la evidencia empírica previa que sugería un efecto positivo de la vía de desarrollo interna sobre el desempeño innovador (Love y Roper, 1999; Frenz e Ietto-Gillies, 2009). Por lo que a la variable SOLO EXTERNA se refiere, los resultados son menos sensibles a las medidas de desempeño utilizadas que en el caso anterior. De hecho se confirma un efecto negativo y significativo de esta vía sobre el desempeño innovador para todas las medidas de desempeño utilizadas excepto INNOPROC, para la que se confirma un efecto positivo y significativo. Este último resultado está en la línea de otros trabajos empíricos 
previos (Love y Roper, 1999; Stuart, 2000; Becker y Dietz, 2002; Francois et al., 2002; Chang, 2003; Rogers, 2004; Faems et al., 2005; Frenz e Ietto-Gillies, 2009) cuyos resultados sugieren que la vía externa es adecuada para acceder al conocimiento especializado no disponible internamente, y permite reducir los costes y riesgos asociados al desarrollo interno. Continuando por la vía INTERNA\&EXTERNA, su efecto positivo y significativo sólo se confirma para la variable INNO EMPRESA, lo que al igual en trabajos previos confirma el efecto positivo de la combinación de ambas vías (Cassiman y Veugelers, 2002; 2006; Beneito, 2006). En cuanto a la vía INTERNA\&COOP, en este caso, se confirma estadísticamente un efecto negativo de la combinación de la vía interna y la cooperación para las variables de desempeño EFECTO e INNOPROC. Por otro lado, se confirma el efecto positivo de su combinación en términos estadísticos para las variables INNO MERCADO, e INNOPROD, en la misma línea de la evidencia empírica obtenida en trabajos previos como los de Becker y Peters (2000) o Schmiedeberg (2008). Continuando con la variable EXTERNA\&COOP se confirma un efecto mayoritariamente negativo y significativo de esta vía sobre el desempeño innovador para las diferentes medidas de desempeño utilizadas, lo que refuerza el argumento de que el conocimiento procedente del exterior de la empresa no puede convertirse en input si ésta no desarrolla internamente sus propias actividades de innovación (Cohen y Levinthal, 1990; Rosenberg, 1990; Kamien y Zang, 2000). La única excepción es para la variable INNOPROC, para la que se confirma un efecto positivo y significativo. Estos resultados son muy similares a los obtenidos con el uso de la vía externa en exclusiva. En último lugar, la variable INTERNA\&EXTERNA\&COOP es la que parece menos sensible a las medidas de desempeño en cuanto a su efecto sobre las mismas. En este caso se confirma el efecto positivo de la combinación de las tres vías, para el conjunto de medidas de desempeño innovador consideradas, con la excepción de la variable INNO EMPRESA, para la que el efecto no es estadísticamente significativo. Este resultado concuerda con trabajos previos (Cassiman y Veugelers, 2002). Además, si se observan los coeficientes de las regresiones MCO (tabla 4), así como los efectos marginales de las regresiones logit (tabla 5), se aprecia que para todas las medidas de desempeño innovador utilizadas la vía cuyo impacto es mayor sobre las mismas es la que combina la interna con la externa y la cooperación. Por tanto, esta vía para el desarrollo de innovaciones no sólo tiene un efecto mayoritariamente positivo sobre el conjunto de medidas de desempeño analizadas, sino que además, su impacto es mayor que el del resto. 
Tabla 4

\section{Regresiones MCO sobre INNO MERCADO/INNO EMPRESA/EFECTO}

\begin{tabular}{|c|c|c|c|c|c|c|}
\hline Variables independientes & $\begin{array}{c}\text { INNO } \\
\text { MERCADO }\end{array}$ & $\begin{array}{c}\text { INNO } \\
\text { MERCADO } \\
\text { (Heckman) }\end{array}$ & $\begin{array}{c}\text { INNO } \\
\text { EMPRESA }\end{array}$ & $\begin{array}{c}\text { INNO } \\
\text { EMPRESA } \\
\text { (Heckman) }\end{array}$ & EFECTO & $\begin{array}{l}\text { EFECTO } \\
\text { (Heckman) }\end{array}$ \\
\hline SOLO INTERNA & $0,84(1,34)$ & $0,38(1,52)$ & $0,97(1,45)$ & $0,52(1,66)$ & $-0,19(0,04) * * *$ & $-0,19(0,04)^{* * * *}$ \\
\hline SOLO EXTERNA & $\begin{array}{c}-5,75 \\
(1,65)^{* * *}\end{array}$ & $\begin{array}{c}-5,52 \\
(1,89)^{* * * *}\end{array}$ & $\begin{array}{c}-4,23 \\
(1,78)^{* * *}\end{array}$ & $\begin{array}{c}-5,02 \\
(2,06)^{* * *}\end{array}$ & $-0,45(0,04) * * *$ & $-0,41(0,05)^{* * *}$ \\
\hline INTERNA\&EXTERNA & $1,76(1,36)$ & $1,56(1,54)$ & $2,17(1,46)^{*}$ & $0,78(1,68)^{*}$ & $-0,04(0,04)$ & $-0,07(0,04)$ \\
\hline INTERNA\&COOP & $3,54(1,57)^{* * *}$ & $3,53(1,78)^{* *}$ & $0,95(1,69)$ & $0,31(1,95)$ & $-0,11(0,04)^{* *}$ & $-0,12(0,05)^{* *}$ \\
\hline EXTERNA\&COOP & $-3,48(2,43)$ & $-5,38(2,82)^{*}$ & $1,65(2,63)$ & $-0,61(3,08)$ & $-0,24(0,07) * * *$ & $\begin{array}{c}-0,21 \\
(0,075)^{* * *}\end{array}$ \\
\hline $\begin{array}{l}\text { INTERNA\& } \\
\text { EXTERNA\&COOP }\end{array}$ & $4,41(1,35)^{* * *}$ & $\begin{array}{c}3,96 \\
(1,55)^{* * *}\end{array}$ & $1,70(1,46)$ & $0,85(1,68)$ & $0,12(0,04)^{* * *}$ & $0,11(0,04)^{* * *}$ \\
\hline TAMAÑO & $\begin{array}{c}1,04 * 10^{-4} \\
\left(2,19 * 10^{-4}\right)\end{array}$ & $\begin{array}{l}1,37 * 10^{-4} \\
\left(2,93 * 10^{-4}\right)\end{array}$ & $\begin{array}{l}-3,27 * 10^{-5} \\
\left(2,36 * 10^{-4}\right)\end{array}$ & $\begin{array}{l}-2,33 * 10^{-5} \\
\left(3,19 * 10^{-4}\right)\end{array}$ & $\begin{array}{l}-3,20 * 10^{-6} \\
\left(6,04 * 10^{-6}\right)\end{array}$ & $\begin{array}{c}2,35^{*} 10^{-6} \\
\left(8,43 * 10^{-6}\right)\end{array}$ \\
\hline CAS1 & $2,89(2,50)$ & $5,78(2,66)^{* *}$ & $-1,02(2,69)$ & $-0,46(2,91)$ & $0,02(0,07)$ & $-0,06(0,07)$ \\
\hline CAS2 & $4,65(2,56)^{*}$ & $3,53(2,78)$ & $2,67(2,76)$ & $1,82(3,04)$ & $0,20(0,07)^{* * *}$ & $-0,115(0,07)$ \\
\hline CAS3 & $0,03(2,59)$ & $-1,18(2,84)$ & $1,71(2,79)$ & $0,68(3,10)$ & $0,28(0,07)^{* * *}$ & $-0,05(0,08)$ \\
\hline CAS4 & $3,27(2,53)$ & $2,15(2,72)$ & $0,31(2,73)$ & $-0,35(2,96)$ & $0,19(0,07)^{* * *}$ & $-0,09(0,07)$ \\
\hline CAS5 & $-2,65(3,03)$ & $3,55(4,51)$ & $-2,01(3,27)$ & $7,77(4,92)$ & $0,25(0,08)^{* * * *}$ & $0,14(0,12)$ \\
\hline CAS6 & $1,66(2,75)$ & $1,43(2,86)$ & $-0,32(2,97)$ & $-0,06(3,12)$ & $0,12(0,07)$ & $0,009(0,08)$ \\
\hline CAS7 & $1,71(2,54)$ & $0,73(2,71)$ & $0,85(2,74)$ & $0,32(2,95)$ & $0,20(0,07)^{* * *}$ & $-0,06(0,07)$ \\
\hline GRUPO & $\begin{array}{c}-1,84 \\
(0,65)^{* * *}\end{array}$ & $\begin{array}{c}-1,95 \\
(0,71)^{* * *}\end{array}$ & $-1,06(0,70)$ & $-0,91(0,77)$ & $0,04(0,02)^{* *}$ & $0,02(0,02)$ \\
\hline MERGEO & $0,58(0,34)^{*}$ & $-0,10(0,45)$ & $0,27(0,36)$ & $-0,25(0,49)$ & $0,06(0,009)^{* * *}$ & $-0,02(0,01)$ \\
\hline REMUSUP & $\begin{array}{c}0,12 \\
(0,135)^{* * * *}\end{array}$ & $\begin{array}{c}0,09 \\
(0,02)^{* * * *}\end{array}$ & $0,02(0,01)^{*}$ & $0,01(0,02)$ & $\begin{array}{l}-3,67 * 10-4 \\
(3,72 * 10-4)\end{array}$ & $\begin{array}{c}-0,004(5 * 10- \\
4)^{* * *}\end{array}$ \\
\hline CONSTANTE & $4,15(2,75)^{*}$ & $\begin{array}{c}9,51 \\
(3,97)^{* * *}\end{array}$ & $\begin{array}{c}12,45 \\
(2,97)^{* * *}\end{array}$ & $\begin{array}{c}16,91 \\
(4,33)^{* * *}\end{array}$ & $2,31(0,07)^{* * * *}$ & $3,20(0,11)^{* * *}$ \\
\hline$\lambda$ & & $-2,64(1,65)$ & & $-2,51(1,81)$ & & $-0,53(0,05) * * *$ \\
\hline Wald $x^{2}$ & & 247,28 **** & & $27,66^{* *}$ & & $351,21 * * *$ \\
\hline $\mathrm{R}^{2}$ & 0,04 & & 0,007 & & 0,0897 & \\
\hline $\mathrm{F}$ & $17,37 * * *$ & & $2,90 * * *$ & & $39,37 * * *$ & \\
\hline
\end{tabular}

$* \mathrm{p}<0,1 \quad * * \mathrm{p}<0,05 * * * \mathrm{p}<0,01$ 
Tabla 5

\section{Regresiones LOGIT sobre INNOPROD/INNOPROC}

\begin{tabular}{|c|c|c|c|c|}
\hline Variables independientes & $\begin{array}{l}\text { INNOPROD } \\
(\mathrm{dy} / \mathrm{dx})\end{array}$ & $\begin{array}{c}\text { INNOPROD } \\
\text { (dy/dx) (Heckman) }\end{array}$ & $\begin{array}{l}\text { INNOPROC } \\
(\mathrm{dy} / \mathrm{dx})\end{array}$ & $\begin{array}{c}\text { INNOPROC } \\
\text { (dy/dx) (Heckman) }\end{array}$ \\
\hline SOLO INTERNA & $-0,02(0,02)$ & $-0,03(0,02)$ & $-0,163(0,02)^{* * *}$ & $-0,16(0,03) * * *$ \\
\hline SOLO EXTERNA & $\begin{array}{c}-0,37 \\
(0,03)^{* * *}\end{array}$ & $-0,36(0,03)^{* * *}$ & $0,09(0,035)^{* * *}$ & $0,07(0,03)^{* *}$ \\
\hline INTERNA\&EXTERNA & $0,02(0,02)$ & $0,009(0,02)$ & $0,02(0,02)$ & $0,02(0,03)$ \\
\hline INTERNA\&COOP & $0,05(0,03)^{* *}$ & $0,02(0,03)^{*}$ & $-0,06(0,03)^{* *}$ & $-0,05(0,03)^{*}$ \\
\hline EXTERNA\&COOP & $\begin{array}{c}-0,19 \\
(0,05)^{* * *}\end{array}$ & $-0,19(0,05)^{* * * *}$ & $0,14(0,04)^{* * *}$ & $0,12(0,05)^{* *}$ \\
\hline $\begin{array}{l}\text { INTERNA\& EXTERNA } \\
\& \text { COOP }\end{array}$ & $0,11(0,02)^{* * *}$ & $0,06(0,03)^{* * *}$ & $0,09(0,02)^{* * *}$ & $0,09(0,03)^{* * *}$ \\
\hline TAMAÑO & $\begin{array}{l}4,18 * 10^{-6} \\
\left(2,12 * 10^{-5}\right)\end{array}$ & $\begin{array}{l}4,25 * 10^{-6} \\
\left(5,09 * 10^{-6}\right)\end{array}$ & $\begin{array}{l}5,42 * 10^{-5} \\
\left(1 * 10^{-5}\right)^{* * *}\end{array}$ & $\begin{array}{c}1,08 * 10^{-5} \\
\left(5,21 * 10^{-6}\right)^{* * *}\end{array}$ \\
\hline CAS1 & $0,09(0,04)^{* *}$ & $0,13(0,05)^{* * *}$ & $-0,09(0,05)^{*}$ & $-0,10(0,05)^{* *}$ \\
\hline CAS2 & $0,18(0,03)^{* * *}$ & $0,09(0,05)^{*}$ & $-0,15(0,06)^{* *}$ & $-0,16(0,05) * * *$ \\
\hline CAS3 & $0,14(0,03)^{* * *}$ & $0,03(0,05)$ & $-0,52(0,26)^{* *}$ & $-0,12(0,05)^{* *}$ \\
\hline CAS4 & $0,09(0,04)^{* *}$ & $-0,001(0,05)$ & $-0,12(0,06)$ & $-0,06(0,05)$ \\
\hline CAS5 & $0,14(0,03)^{* * *}$ & $0,30(0,07)^{* * *}$ & $-0,05(0,05)$ & $-0,15(0,08)^{*}$ \\
\hline CAS6 & $0,06(0,04)$ & $0,03(0,05)$ & $-0,06(0,07)$ & $-0,11(0,05)^{* *}$ \\
\hline CAS7 & $0,09(0,04) * *$ & $0,002(0,05)$ & $-0,11(0,06)$ & $-0,03(0,05)$ \\
\hline GRUPO & $0,006(0,01)$ & $0,006(0,01)$ & $0,03(0,01)^{* * *}$ & $0,04(0,01)^{* * *}$ \\
\hline MERGEO & $\begin{array}{c}0,04 \\
(0,006)^{* * *}\end{array}$ & $0,009(0,008)$ & $0,03(0,006)^{* * * *}$ & $0,03(0,01)^{* * *}$ \\
\hline REMUSUP & $\begin{array}{c}0,0005 \\
(0,0002)^{* *}\end{array}$ & $-0,001(0,0003)^{* * *}$ & $-0,001(0,0002)^{* * *}$ & $-0,002(0,0003)^{* * *}$ \\
\hline$P$ & & $-0,21(0,03)^{* * *}$ & & $-0,01(0,03)$ \\
\hline Wald $x^{2}$ & & $406,61 * * *$ & & $541,39 * * *$ \\
\hline Log likelihood & $-3851,6052$ & & $-3979,9385$ & \\
\hline LR Chi-2 & $664,79 * * *$ & & $600,89 * * *$ & \\
\hline Pseudo $\mathrm{R}_{2}$ & 0,0794 & & 0,0702 & \\
\hline
\end{tabular}

Por último, presentamos un resumen de los principales resultados relativos a las variables de control introducidas en el análisis.

Comenzando con la variable TAMAÑO, se confirma su efecto positivo y significativo sobre el desempeño innovador para INNOPROC. Continuando con las variables de sector, se confirma que el sector puede tener una cierta influencia sobre la innovación en producto (INNOPROD) o en proceso (INNOPROC). En cuanto a la pertenencia a un grupo (GRUPO) los resultados no son concluyentes. El efecto es positivo sobre el desempeño inno- 
vador para el caso de EFECTO e INNOPROC. Por otro lado, los resultados muestran un efecto negativo para INNO MERCADO. La amplitud del mercado geográfico (MERGEO) por su parte tiene un efecto positivo sobre INNO MERCADO, EFECTO, INNOPROD e INNOPROC. En último lugar, los resultados sugieren un efecto positivo de la existencia de personal con estudios superiores (REMUSUP) sobre las variables INNO MERCADO e INNO EMPRESA, mientras que para INNOPROD, INNOPROC y EFECTO el signo es negativo y significativo.

La tabla 6 recoge la media de la intensidad de los impactos de cada una de las vías para el desarrollo de innovaciones (interna, externa o cooperación), sobre las cinco medidas de desempeño utilizadas. Los valores medios de los impactos se han calculado como la media de los impactos estadísticamente significativos de cada una de las vías en todas sus posibles formas de uso (sola o combinada con el resto de vías) sobre el desempeño innovador. Comenzando con la vía interna, los datos de la tabla 6 sugieren que el impacto positivo medio de esta vía es mayor para el desarrollo de innovaciones para el mercado, seguido del desarrollo de innovaciones de mercado, y un impacto bajo para el desarrollo de innovaciones de producto. Por otro lado, aunque bajos, se tienen impactos medios negativos de la vía interna sobre las innovaciones medidas en términos de sus efectos y las innovaciones de proceso.

Respecto de la vía externa, la tabla 6 muestra que ésta solo tiene un impacto positivo medio para el desarrollo de innovaciones de proceso. En cuanto a sus efectos negativos, estos son mayores para el desarrollo de innovaciones para el mercado y la empresa, y menores cuando las innovaciones se miden a través de sus impactos o de la introducción de innovaciones de producto.

Por último, en relación a la vía cooperación, su mayor impacto positivo medio se tiene para el desarrollo de innovaciones para el mercado. Ésta vía, tiene también un impacto positivo medio para las innovaciones de proceso, pero de menor importancia. En cuanto a sus impactos negativos medios, éstos se tienen principalmente para el desarrollo de innovaciones cuando se miden a través de sus efectos, en primer lugar, y de producto en segundo. Finalmente, la vía cooperación no tiene impacto en el desarrollo de innovaciones para la empresa.

Tabla 6

Impactos medios de cada vía de innovación sobre cada medida de desempeño innovador

\begin{tabular}{|c|c|c|c|c|c|c|c|c|c|c|}
\hline & $\begin{array}{l}\text { INNO } \\
\text { MER- } \\
\text { CADO }\end{array}$ & $\begin{array}{c}\text { INNO } \\
\text { MER- } \\
\text { CADO } \\
\text { (Heck- } \\
\text { man) } \\
\end{array}$ & $\begin{array}{l}\text { INNO } \\
\text { EMPRE- } \\
\text { SA }\end{array}$ & $\begin{array}{c}\text { INNO } \\
\text { EM- } \\
\text { PRESA } \\
\text { (Heck- } \\
\text { man) }\end{array}$ & EFECTO & $\begin{array}{c}\text { EFECTO } \\
\text { (Heck- } \\
\text { man) }\end{array}$ & $\begin{array}{l}\text { INNO- } \\
\text { PROD }\end{array}$ & $\begin{array}{c}\text { INNO- } \\
\text { PROD } \\
\text { (Heck- } \\
\text { man) }\end{array}$ & $\begin{array}{l}\text { INNO- } \\
\text { PROC }\end{array}$ & $\begin{array}{c}\text { INNO- } \\
\text { PROC } \\
\text { (Heck- } \\
\text { man) }\end{array}$ \\
\hline $\begin{array}{l}\text { Impacto medio de } \\
\text { la vía INTERNA }\end{array}$ & 1,99 & 1,87 & 0,54 & 0,20 & $-0,05$ & $-0,05$ & 0,04 & 0,02 & $-0,03$ & $-0,03$ \\
\hline $\begin{array}{l}\text { Impacto medio de } \\
\text { la vía EXTERNA }\end{array}$ & $-0,34$ & $-1,74$ & $-1,06$ & $-1,26$ & $-0,14$ & $-0,13$ & $-0,11$ & $-0,12$ & 0,08 & 0,07 \\
\hline $\begin{array}{l}\text { Impacto medio de } \\
\text { la vía COOPE- } \\
\text { RACIÓN }\end{array}$ & 2,65 & 0,70 & $\begin{array}{l}\text { Sin im- } \\
\text { pacto }\end{array}$ & $\begin{array}{l}\text { Sin im- } \\
\text { pacto }\end{array}$ & $-0,08$ & $-0,07$ & $-0,01$ & $-0,04$ & 0,06 & 0,05 \\
\hline
\end{tabular}




\section{CONCLUSIONES}

El presente trabajo se ha planteado el estudio del efecto sobre el desempeño innovador de las diferentes vías elegidas por las empresas para el desarrollo de sus actividades de innovación. Para la realización del estudio empírico se ha utilizado una muestra integrada por 6.807 empresas activas en innovación. El criterio elegido para la selección de la muestra (ser o no activa en innovación) se justifica por la necesidad de incorporar en el estudio a las empresas que desarrollan algún tipo de actividad innovadora con independencia de los resultados que hayan obtenido con las mismas.

En cuanto a los análisis realizados sugieren en primer lugar que la utilización de una única vía, ya sea interna o externa, para la realización de actividades innovadoras tiene un efecto mayoritariamente negativo sobre el desempeño innovador. Por lo tanto, parece que las empresas deben recurrir a la utilización conjunta de diferentes vías para obtener mejores resultados. Por lo que al uso de la vía interna de manera exclusiva se refiere, estos resultados dan apoyo a los argumentos procedentes del modelo de innovación abierta ("open innovation") que sugieren que las ventajas derivadas de la elección de la vía interna para el desarrollo de actividades de I+D se han visto reducidas como consecuencia de la mayor dificultad de apropiación de los resultados de innovación debido a la mayor movilidad de los trabajadores (Chesbrough, 2003a; 2003b; Laursen y Salter, 2006). En el caso de la vía externa, este resultado sugiere por otro lado, que en línea con los supuestos procedentes del concepto de capacidad de absorción, según los que el conocimiento procedente del exterior de la empresa no puede convertirse en un input para los procesos innovadores si ésta no desarrolla internamente su propia investigación que le permita absorber dicho conocimiento (Cohen y Levinthal, 1990). Por último, parece que la utilización de la vía externa en exclusiva favorece el desarrollo de innovaciones de proceso, por lo tanto, parece que si bien el uso de la vía externa de manera exclusiva redunda en un menor desempeño innovador en términos generales, supone un mayor desempeño en términos de innovaciones de proceso.

En el caso de la vía interna, su combinación con la vía externa supone mejores resultados en términos de innovaciones para la empresa, mientras su combinación con la cooperación redundará en un mejor desempeño en términos de innovaciones de producto, y dentro de éstas, para las que suponen una novedad en el mercado. Estos resultados están en la línea de los supuestos procedentes del enfoque de la capacidad de absorción así como con trabajos empíricos previos (Becker y Peters, 2000; Beneito, 2006; Cassiman y Veugelers, 2002; 2006; Schmiedeberg, 2008). Sin embargo, la combinación de la vía interna con la cooperación reduce el desempeño innovador en términos de sus efectos y de innovación de procesos, un resultado que confirma algunas de las propuestas formuladas desde el campo de la Organización Industrial y contrastadas empíricamente por algunos autores (Veugelers, 1997; Jirjahn y Kraft, 2006). Según este punto de vista, la combinación de la cooperación y la vía interna puede tener un efecto negativo sobre el desempeño innovador en presencia de spillovers de salida, al dificultar la apropiación y el control de los resultados de la I+D desarrollada en cooperación por parte de la empresa, lo que implicaría un menor desempeño innovador (Laursen y Salter, 2006), lo que en este trabajo se confirma exclusivamente para el desempeño en términos de sus efectos e innovaciones de procesos.

Respecto de la vía externa, queda comentar los resultados obtenidos para su combinación con la cooperación. En este caso, su efecto sobre el desempeño innovador es mayo- 
ritariamente negativo. En concreto, la combinación de la vía externa con la cooperación implica menor desempeño innovador en términos de sus efectos y de innovaciones de producto, resultado que apoya la noción de capacidad de absorción (Cohen y Levinthal, 1990). Por otro lado, la vía que combina la externa con la cooperación genera un mayor desempeño innovador de procesos. Este resultado sugiere que para el desarrollo de innovaciones de proceso el conocimiento procedente del exterior de la empresa constituye un input muy importante, y en éste caso no es necesario el desarrollo interno que permita la absorción del conocimiento procedente del exterior, tal y como sugería el concepto de capacidad de absorción (Cohen y Levinthal, 1990).

Por último, en cuanto a la combinación de las tres vías, esta alternativa permite alcanzar los mejores resultados en términos de rendimiento. Una vez más, éste resultado estaría en línea con los planteamientos realizados desde la perspectiva de la capacidad de absorción, en el sentido de que únicamente las empresas que disponen de suficiente conocimiento interno están en disposición de aprovechar mejor los conocimientos procedentes del exterior de la empresa y la cooperación.

En cuanto a la existencia de posibles diferencias en los resultados obtenidos en función de las medidas de performance utilizadas, las vías cuyo efecto sobre el desempeño parece más independiente de la medida utilizada son sólo externa, interna\&externa, externa\&cooperación e interna,externa\&cooperación. El resto de vías analizadas muestran resultados que difieren en mayor medida en función de las medidas de desempeño utilizadas, lo que indicaría que la elección de las vías más adecuadas para la realización de actividades innovadoras deberá tener en cuenta cuáles son los objetivos que se persiguen. En el caso de la vía interna, los resultados sugieren que su utilización en exclusiva disminuye el rendimiento en términos de sus efectos y de innovaciones de proceso pero no tiene efectos sobre el resto de medidas de desempeño. Por lo que se refiere a la vía interna y cooperación, los resultados indican un efecto negativo cuando las medidas de desempeño se centran la medición de los efectos, y la introducción de innovaciones de procesos, mientras que esta combinación puede tener un efecto potenciador para el desarrollo de innovaciones de producto, y dentro de estas sobre todo, aquellas que suponen una novedad para el mercado. Por último, la utilización de la vía externa conjuntamente con la cooperación parece tener un efecto positivo cuando el objetivo se mide en términos de innovaciones en proceso y negativo en el resto.

Por último, si prestamos atención al impacto medio de las vías interna, externa y cooperación se pueden extraer las siguientes conclusiones. Comenzando con la vía interna, los resultados sugieren que se trata de una vía adecuada para la obtención de mejor desempeño en términos de la introducción de innovaciones de producto, tanto si suponen una novedad para el mercado como para la empresa, mientras que implican una disminución del desempeño innovador en términos de sus efectos y las innovaciones de proceso. Por tanto, estos resultados dan apoyo a los supuestos procedentes del enfoque de innovación abierta ("open innovation”), que destacan el alcance limitado de la vía interna como fuente de crecimiento del desempeño innovador pero solo para el caso de que éste se mida en términos de sus efectos o de innovaciones de proceso. Continuando con la vía externa, su impacto medio sobre el desempeño innovador es negativo, con la excepción del desarrollo de innovaciones de proceso. Es decir, este resultado apoya los argumentos presentes en la literatura previa de que la vía externa es adecuada para acceder al conocimiento especializado no dispo- 
nible internamente, explotar economías de escala asociadas a la especialización, así como reducir los costes y riesgos del desarrollo interno, pero solo cuando persigue el desarrollo de innovaciones de proceso. Por último, respecto de la cooperación, nuestros resultados sugieren que en media, se trata de una vía que redunda en mayores resultados innovadores en términos de innovaciones de producto para el mercado y de proceso, mientras que por el contrario, puede disminuir el desempeño innovador en cuanto a los efectos de las innovaciones y la innovación de producto en general. Por otro lado, esta vía no tiene impacto medio para el desarrollo de innovaciones que suponen una novedad para la empresa. Estos resultados dan apoyo empírico a los argumentos teóricos que proponían las ventajas de la cooperación para el desarrollo de innovaciones como consecuencia de la división del trabajo, el acceso a conocimiento externo disponible, el reparto de costes y riesgos, el acceso a financiación pública o el mejor control de los spillovers de salida, pero solo para el caso del desarrollo de innovaciones de producto para el mercado o de proceso, no tratándose de la vía más adecuada, en media, para el resto de medidas de desempeño.

Implicaciones del estudio

A la vista de las conclusiones anteriores parece que en un sentido práctico, los resultados de este trabajo nos llevan a afirmar que los directivos deben tener en cuenta a la hora de diseñar su estrategia en materia de innovación cuales son los objetivos que se persiguen de manera prioritaria con la misma, puesto que no todas las vías disponibles para el desarrollo de actividades de innovación tienen los mismos efectos sobre todas las medidas de desempeño innovador, sino que éstos varían considerablemente de unas medidas de desempeño a otras. Siguiendo esta línea, los resultados nos permiten realizar las siguientes recomendaciones para los gerentes de empresas, en cuanto a la vía o vías más adecuadas para sus empresas en función del objetivo que persiguen, esto es, del tipo de desempeño innovador que desean alcanzar. En primer lugar, se recomienda a aquellas empresas que persiguen el desarrollo de innovaciones de producto optar por la vía interna, siempre que sea combinada con la cooperación, y con la externa y la cooperación simultáneamente. Dentro de las innovaciones de producto, aquellas empresas que quieran desarrollar innovaciones que supongan una novedad en el mercado deberán optar por las vías interna y cooperación, siempre que la interna se combine bien con la cooperación o con las vías externa y cooperación simultáneamente. Respecto de las empresas que persigan el desarrollo de innovaciones de producto para la empresa, deberán optar por la vía interna combinándola con la externa. Continuando con las empresas cuyo objetivo sea mejorar los efectos de sus innovaciones, deberán apostar por la vía que combina la interna con la externa y la cooperación. Por último, aquellas empresas que buscan mayor desempeño innovador de procesos, deben apoyarse principalmente en las vías externa y cooperación, tanto la vía externa de manera exclusiva, como combinada con la cooperación o con la interna y la cooperación.

El estudio también revela que la estrategia seguida con mayor frecuencia por las empresas integrantes de la muestra (solo interna, 26,34\% del total) tiene un efecto mayoritariamente negativo sobre el desempeño innovador. No obstante, este efecto se transforma en positivo para algunas de las medidas de desempeño cuando la vía interna se combina con cualquiera de las restantes. En este sentido, una recomendación que puede hacerse a las empresas es la de ampliar el abanico de vías para el desarrollo de actividades de innovación, en la línea de lo propuesto en los modelos de "open innovation". 
En cuanto a las limitaciones del trabajo, comentar que algunos de los resultados obtenidos en cuanto al efecto de las vías para el desarrollo de actividades de innovación sobre el desempeño innovador pueden deberse a la no consideración del efecto temporal. Esto es que en algunos casos las actividades innovadoras pueden no generar efectos sobre el desempeño en el periodo corriente, y presentarse con un retardo temporal, consideración ésta que podría ser tenida en cuenta para el desarrollo de trabajos futuros. Por otro lado, la forma en que se han construido las variables, en particular las relativas a las diferentes vías de innovación mediante dummys puede haber condicionado los resultados obtenidos. En este sentido, podría ser interesante de cara a futuras investigaciones utilizar metodologías adicionales de medición que permitiesen la comparación con los resultados aquí obtenidos. Por último, sería también relevante profundizar en la identificación y análisis de variables contextuales que puedan contribuir a explicar los resultados obtenidos, como pueden ser el tipo e importancia de los spillovers, o las medidas de apropiabilidad de los resultados de innovación.

\section{REFERENCIAS BIBLIOGRÁFICAS}

ABERNATHY, W. J. y CLARK, K. B. (1985): "Innovation: Mapping the winds of creative destruction", Research Policy, Vol. 14, pp. 3-22.

ARANGO LONDOÑO, J. F. (2012): "La gestión de la innovación como la gestión de un ecosistema heterogéneo y estructurado", Cuadernos de Gestión, Vol. 12. Especial Innovación, pp. 125-137.

ARORA, A. y GAMBARDELLA, A. (1994): "Evaluating technological information and utilizing it: scientific knowledge, technological capability and external linkages in biotechnology", Journal of Economic Behavior and Organization, Vol. 24, pp. 91-114.

BAUMOL, W.J, (2002): The Free-Market Innovation Machine, Princeton, Princeton University Press.

BECKER, W. y DIETZ, J. (2002): "R\&D Cooperation and Innovation Activities of Firms-Evidence for the German Manufacturing Industry", Volkswirtschaftliche Diskussionsreihe der Universität Augsburg, No. 222.

BECKER, W. y PETERS, J. (2000): "Technological opportunities, absorptive capacity and innovation", Discussion Paper series, Universitaet Ausburg, No. 195.

BELDERBOS, R.; CARREE, M. y LOKSHIN, B. (2004a): "Cooperative R\&D and firm performance", Research Policy, Vol. 33, No. 10, pp. 1477-1492.

BELDERBOS, R.; CARREE, M.; DIEDEREN, B.; LOKSHIN, B. y VEUGELERS, R. (2004b): "Heterogeneity in R\&D cooperation strategies", International Journal of Industrial Organization, Vol. 22, No. 8-9, pp. 1237-1263.

BENEITO, P. (2003): "Choosing among alternative technological strategies: an empirical analysis of formal sources of innovation", Research Policy, Vol. 32, pp. 693-713.

BENEITO, P. (2006): "The innovative performance of in-house and contracted R\&D in terms of patents and utility models", Research Policy, Vol. 35 N.4, pp. 502-517.

BOUGRAIN, F. y HAUDEVILLE, B. (2002): "Innovation, collaboration and SME's internal research capacities", Research Policy, Vol. 31, pp. 735-747.

BROCKHOFF, K. (1992): "R\&D COOPERATION BETWEEN FIRMS - A PERCEIVED TRANSACTION COST PERSPECTIVE”, Management Science, Vol. 38, No. 4, pp. 514-524.

CALOGHIROU, Y.; KASTELLI, I. y TSAKANIKAS, A. (2004): “Internal and external knowledge sources: complements or substitutes for innovative performance?, Technovation, Vol.24, №. 1 , pp. 29-39. 
CAPON, N.; FARLEY, J.U. y HOENIG, S. (1990): "Determinants of financial performance: a metaanalysis", Management Science, Vol. 36, pp. 1143-1159.

CASSIMAN, B. y VEUGELERS, R. (2002): "R\&D cooperation and spilllovers: some empirical evidence from Belgium", American Economic Review, Vol. 29, Nº. 4, pp. 1169-1184.

CASSIMAN, B. y VEUGELERS, R. (2006): "In Search of Complementarity in Innovation Strategy", Management Science, Vol. 52, No.1, pp. 68-82.

CASTAÑO MARTÍNEZ, M. S. (2012): "Innovación y política tecnológica: el caso del sector transformador y el sector servicios a empresas", Cuadernos de Gestión, Vol. 12. Especial Innovación, pp. 59-72.

CASTELLACCI, F. (2008): "Technological paradigms, regimes and trajectories: Manufacturing and service in a new taxonomy of pectoral patterns of innovation", Research Policy, Vol. 37, №. 6-7, pp. 978-994.

CETINDAMAR, D. y ULUSOY, G. (2008); "Innovation performance and partnerships in manufacturing firms in Turkey", Journal of Manufacturing Technology Management, Vol. 19, №. 3 , pp. 332-360.

CHANG, Y-C. (2003): "Benefits of Co-operation on innovative performance: evidence from integrated circuits and biotechnology firms in the UK and Taiwan", $R \& D$ Management, Vol. 33, $\mathrm{N}^{\circ}$. 4, pp. 425-437.

CHEN, Y. y YUAN, Y. (2007): "The innovation strategy of firms: empirical evidence from the Chinese high-tech industry", Journal of Technology Management, Vol. 2, №. 2, pp.145-153.

CHESBROUGH, H.W. (2003a): Open Innovation, Boston, MA, Harvard Business School Press.

CHESBROUGH, H.W. (2003b): "The era of open innovation”, Sloan Management Review Summer, pp. 35-41.

COASE, R. (1937): "The nature of the Firm", Economica, Vol. 4, pp. 386-405

COCKBURN, I.M.; MACGARVIE, M. y MULLER, E. (2008): "Patent thickets, licensing and innovative performance", ZEW Discussion paper, $\mathrm{N}^{\circ} .2009-7$.

COHEN, W. y LEVINTHAL, D. (1990): "Absorptive capacity: a new perspective on learning and innovation", Administrative Science Quarterly, Vol. 35, №. 1, pp. 128-152.

CRISCUOLO, C. y HASKEL, J. (2003): "Innovation and Productivity Growth in the UK: Evidence from CIS2 and CIS3", Working Paper, Centre for Research into Business Activity.

CZARNITKI, D. y WASTYN, A. (2009): "Does professional knowledge management improve innovation performance at the firm level?" ZEW Discussion paper No. 09-067.

FAEMS, D.; BART VAN LOOY, B. y DEBACKERE. K. (2005): "Interorganizational Collaboration and Innovation: Toward a Portfolio Approach", The Journal of Product Innovation Management, Vol. 22, $\mathrm{N}^{\circ} .3$, pp. 238-250.

FAGERBERG, J. (2005): "Innovation: A guide to the literature", The Oxford Handbook on Innovation, Oxford, Oxford University Press, pp. 1-27.

FOSFURI, A. y TRIBÓ, J.A. (2008); "Exploring the antecedents of potential absorptive capacity and its impact on innovation performance", Omega, Vol. 36, №. 2, pp. 173-187.

FREEMAN, C. (1991): "Networks of innovators: A synthesis of research issues", Research Policy, Vol. 20, No. 5, pp. 499-514.

FRANCOIS, J.P.; FAVRE, F. y NEGASSI, S. (2002): "Competence and organization: two drivers of innovation", Economics of Innovation and New Technology, Vol. 11, pp. 249-270.

FRENZ, M. y IETTO-GILLES, G. (2009): "The impact on innovation performance of different sources of knowledge: evidence from the UK Community Innovation Survey“, Research Policy, Vol. 38, No.7, pp. 1125-1135.

FRITSCH, M. (2003): "Does R\&D-cooperation behaviour differ between regions?", Industry and Innovation, Vol. 10, $\mathrm{N}^{\circ} .1$, pp. 25-39. 
GALINDO MARTÍN, M-A.; RIBEIRO, D. y MÁNDEZ PICAZO, M. T. (2012): "Innovación y crecimiento económico: factores que estimulan la innovación", Cuadernos de Gestión, Vol. 12. Especial Innovación, pp. 51-58.

HAOUR, G. (1992): "Stretching the knowledge-base of the enterprise through contract research", $R \& D$ Management, Vol. 22, No .2 , pp. 177-182.

HART, S. (1992): “An integrative framework for strategy-making process", Academy of Management Review, Vol. 17, pp. 327-331.

HARTUNG, V. y MACPHERSON, A. (2000): "Innovation and collaboration in the geographic information systems (GIS) industry: evidence from Canada and the United States", $R \& D$ Management, Vol. 30, No. 3, pp. 225-234.

HECKMAN, J. (1979): "Sample selection bias as a specification error", Econometrica, Vol. 47, pp. 1219-1240.

HUANG, Y-A.; CHUNG, H-J. y LIN, C. (2009): "R\&D SOURCING STRATEGIES: DETERMINANTS AND CONSEQUENCES", Technovation, Vol. 29, Nº. 3, pp. 155-169.

JANTUNEN, A. (2005): "Knowledge-processing capabilities and innovative performance: an empirical study", European Journal of Innovation Management, Vol. 8, №. 3, pp. 336-349.

JIRJAHN, U. y KRAFT, K. (2006): "Do spillovers stimulate incremental or drastic product innovations? Hypotheses and evidence from German establishment data", ZEW Discussion paper, $\mathrm{N}^{\circ}$. 06-023.

KAMIEN, M. y ZANG, I. (2000): "Meet me halfway: research joint ventures and absorptive capacity", International Journal of Industrial Organization, Vol. 18, №. 7, pp. 995-1012.

KATILA, R. (2002): "New product search over time: past ideas in their prime?", Academy of Management Journal, Vol. 45, pp. 995-1010.

KATILA, R. y AHUJA, G. (2002): "Something old, something new: a longitudinal study of search behaviour and new product introduction", Academy of Management Journal, Vol. 45, №. 8, pp. 1183-1194.

KATZ, R. y ALLEN, T.J. (1982), "Investigating the Not Invented Here (NIH) Syndrome: A Look at the Performance, Tenure, and Communication Patterns of 50 R\&D Project Groups", $R \& D$ Management, Vol. 12, $\mathrm{N}^{\circ}$. 1, pp. 7-21.

KIRNER, E.; KINKEL, S. y JAEGER, A. (2009): "Innovation paths and the innovation performance of low-technology firms-An empirical analysis of German industry", Research Policy, Vol. $38, N^{\circ} .3$, pp. 447-458.

KLOMP, L. y VAN LEEWEN, G. (2001): "Linking Innovation and Firm Performance: A New Approach", International Journal of the Economics of Business, Vol. 8, No. 3, pp 343-364.

KNOBEN, J. (2009): "Localized inter-organizational linkages, agglomeration effects, and the innovative performance of firms", Annals of Regional Science, Vol. 43, No. 3, pp. 757-779.

LAURSEN, K. y SALTER, A. (2006): "Open for innovation: the role of openness in explaining innovation performance among U.K. manufacturing firms", Strategic Management Journal, Vol. $27, \mathrm{~N}^{\circ} .2$, pp. 131-150.

LEIPONEN, A. (2005): "Organization of Knowledge and Innovation: The Case of Finnish Business Services", Industry and Innovation, Vol. 12, N. 2, pp. 185-203.

LI, H. y ATUAHENE-GIMA, K. (2001): "Product innovation strategy and performance of new technology ventures in China", Academy of Management Journal, Vol. 44, pp. 1123-1134.

LÓPEZ, A. (2008): "Determinants of R\&D cooperation: Evidence from Spanish manufacturing firms", International Journal of Industrial Organization, Vol. 26, No. 1, pp. 113-136.

LOKSHIN, B.; VAN GILS, A. y BAUER, E. (2009): "Crafting firm competencies to improve innovative performance", European Management Journal, Vol. 27, pp. 187-196.

LOVE, J.H y ROPER, S. (1999): "R\&D, technology transfer and networking effects on innovation intensity", Review of Industrial Organization, Vol. 15, N. 1, pp. 43-64. 
LOVE, J. H. y ROPER, S. (2001), "Location and network effects on innovation success: evidence from UK, German and Irish manufacturing plants", Research Policy, Vol. 30, No. 4, pp. 643-661.

MARSILI, O. y SALTER, A. (2006): “The dark matter of innovation: design and innovative performance in Dutch manufacturing", Technology Analysis \& Strategic Management, Vol. 18, No. 5, pp.515-534.

MEEUS, M. T. H. y OERLEMANS, L. A. G. (2000): "Firm behaviour and innovative performance. An empirical exploration of the selection-adaptation debate", Research Policy, Vol. 29, N $^{\circ} .1$, pp. 41-58.

MONJON, S. y WAELBROECK, P. (2003): "Assessing Spillovers from Universities to Firms: Evidence from French Firm-level Data", International Journal of Industrial Organization, Vol. 21, No.9, pp. 1255-1270.

NAVARRO ARANCEGUI, M. (2002): "La cooperación para la innovación en la empresa española desde una perspectiva internacional comparada", Economía Industrial, №. 344, pp. 47-66.

PEREIRA, A. y VENCE, X. (2012): Factores empresariales clave para la eco-innovación: una revisión de estudios empíricos recientes a nivel de empresa, Cuadernos de Gestión, Vol. 12. Especial Innovación, pp. 73-103.

PISANO, G. (1990): "The R\&D boundaries of the firm: An empirical analysis", Administrative Science Quarterly, Vol. 35, pp. 153-176.

RIGBY, D. y ZOOK, C. (2002): “Open-Market Innovation”, Harvard Business Review, Vol. 80, N. 10 , pp. 80-89.

ROGERS, M. (2004): “Networks, firm size and innovation”, Small Business Economics, Vol. 22, Nº. 2, pp. 141-153.

ROSENBERG, N. (1990): "Why do firms do basic research (with their own money)?", Research Policy, Vol. 19, pp. 165-174.

SAVIOTTI, P. P. y METCALFE, J. S. (1984): “A theoretical approach to the construction of technological output indicators", Research Policy, Vol. 13, pp. 141-151.

SCHMIEDEBERG, C. (2008): "Complementarities of innovation activities: An empirical analysis of the German manufacturing sector", Research Policy, Vol.37, №. 9, pp. 1492-1503.

SCHUMPETER, J.A. (1939): Business Cycles: A Theoretical, Historical and statistical Analysis of the Capitalist Process, New York, McGraw-Hill.

SOUITARIS, V. (2002): "Firm-specific competencies determining technological innovation: a survey in Greece", R\&D Management, Vol. 32, No. 1, pp. 61-77.

STUART, T.E. (2000): "Interorganizational alliances and the performance of firms: a study of growth and innovation rates in a high-technology industry", Strategic Management Journal, Vol. 21, No. 8, pp. 791-811.

TEECE, D. (1986): "Profiting from technological innovation: Implication for integration, collaboration, licensing and public policy", Research Policy, Vol.15, pp. 285-305.

TETHER, B. (2005): "Do services innovate (differently)? Insights from the European Innobarometer Survey", Industry and Innovation, Vol. 12, No. 2, pp. 153-184.

TSAI, K-H. (2009): "Collaborative networks and product innovation performance: toward a contingency perspective”, Research Policy, Vol. 38, №. 85, pp. 765-778.

TSAI, K-H. y WANG, J-C. (2009): "External technology sourcing and innovation performance in LMT sectors: an analysis on the Taiwanese Technological Innovation Survey“, Research Policy, Vol. 38, No. 3, pp. 518-526.

URIEL JIMÉNEZ, E. y ALDÁS MANZANO, J. (2005): Análisis multivariante aplicado: aplicaciones al marketing, investigación de mercados, economía, dirección de empresas y turismo, Madrid, Thomson.

VEGA-JURADO, J.; GUTIERREZ-GRACIA, A. y FERNANDEZ-DE-LUCIO, I. (2009): "Does external knowledge sourcing matter for innovation? Evidence from the Spanish manufacturing industry", Industrial and Corporate Change, Vol. 18, №. 4, pp. 637-670. 
VEUGELERS, R. (1997): “Internal R\&D expenditures and external technology sourcing”, Research Policy, Vol. 26, No. 3, pp. 303-315.

VEUGELERS, R. y CASSIMAN, B. (1999): "Make and buy in innovation strategies: Evidence from Belgian manufacturing firms", Research Policy, Vol. 28, N. 1, pp. 285-305.

VEUGELERS, R. y CASSIMAN, B. (2005): "R\&D cooperation between firms and universities. Some empirical evidence from Belgian manufacturing firms", International Journal of Industrial Organization, Vol. 23, N. 5-6, pp. 355-379.

WETERINGS, A. y BOSCHMA, R. (2009): "Does spatial proximity to customers matter for innovative performance? Evidence form the Dutch software sector", Research Policy, Vol. 38, №. 5 , pp.746-755.

WILLIAMSON, O.E., (1985), The Economic Institutions of Capitalism, New York, Free Press.

WOERTER, M. (2009): "Industry diversity and its impact on the innovation performance of firms", Journal of Evolutionary Economics, Vol. 19, №. 5, pp. 675-700.

ZABALA-ITURRIAGAGOITIA, J.M. (2012): "La vigilancia tecnológica como una herramienta para la gestión de la innovación", Cuadernos de Gestión, Vol. 12. Especial Innovación, pp. 105124. 